\title{
ORIGINAL RESEARCH \\ Dural Ectasia in Marfan Syndrome: A Case Control Study
}

\begin{abstract}
R. Lundby
S. Rand-Hendriksen

J.K. Hald

F.G. Lilleås

A.H. Pripp

S. Skaar

B. Paus

O. Geiran

BACKGROUND AND PURPOSE: Dural ectasia (DE) is one of the major criteria of Marfan syndrome (MFS). Our aim was to establish the prevalence of DE in an adult population fulfilling the Ghent criteria for MFS and to assess definitions of DE.

MATERIALS AND METHODS: One hundred five adults with suspected MFS were included. MR imaging at $1.5 \mathrm{~T}$ was performed unless contraindicated; then $\mathrm{CT}$ was obtained. Lumbosacral anteroposterior vertebral body diameters (VBD) and dural sac diameters (DSD) were measured. Dural sac ratios $(\mathrm{DSR}=\mathrm{DSD} / \mathrm{BD})$ at levels $\mathrm{L} 3$ through S1 were calculated. Anterior meningoceles, herniations of nerve root sleeves, and scalloping were characterized. One hundred one sex- and age-matched patients were included as controls.
\end{abstract}

H.-J. Smith

RESULTS: We identified 3 patient groups: 1) fulfilling Ghent criteria independent of DE ( $n=73), 2$ ); fulfilling Ghent criteria dependent on DE $(n=14)$, and 3); and suspected MFS, not fulfilling Ghent criteria $(n=18)$. DE was found in $86 \%$ of group 1 . At levels L4-S1, mean DSRs were significantly higher in group 1 than in group 3 and controls $(P<.001)$. Herniations of the nerve root sleeves were present in $73 \%$ in group 1 versus $1 \%$ in controls. Anterior meningoceles were found in $37 \%$ and $14 \%$ in groups 1 and 2, respectively, but not in group 3 or controls.

CONCLUSIONS: The diagnosis of DE on MR imaging or CT should be based on the presence of at least 1 of the following criteria: anterior meningoceles or nerve root sleeve herniation, DSD at S1 or below larger than DSD at L4, and DSR at S1 >0.59.
$\mathbf{M}$ arfan syndrome (MFS) is an autosomal dominant disorder of connective tissue. Life expectancy is generally reduced in these patients, mainly due to progressive dilation and dissection of the aorta. Other manifestations include dislocation of the lens and skeletal deformities. Early diagnosis, follow-up, and treatment are important to prolong life and reduce disability.

The diagnostic process to identify patients with MFS is challenging because it is based on the Ghent criteria, ${ }^{1,2}$ which requires the assessment of a number of clinical, genetic, and radiologic features. In addition to exploration of the dura mater, the diagnosis is based on findings in the skeletal, ocular, cardiovascular, and pulmonary systems, the skin and integument, a family history, and mutations in the gene encoding for fibrillin 1 (FBN1). Mutations in the FBN1 gene are also found in individuals not fulfilling the Ghent criteria; consequently, the diagnosis is still based on other criteria as well as genetics.

Dural ectasia (DE) is one of the major criteria of MFS in the Ghent nosology and has been defined as "enlargement of the neural canal anywhere along the spinal column, but nearly always in the lower lumbar and sacral regions; thinning of the

Received January 8, 2009; accepted after revision March 11.

From the Department of Radiology (R.L., J.K.H., S.S., H.-J.S.), Section of Biostatistics, Research Services Department (A.H.P.), and Division for Cardiac and Respiratory Medicine and Surgery (0.G.), Oslo University Hospital, Rikshospitalet, Oslo, Norway; TRS National Resource Centre for Rare Disorders (S.R.-H.), Sunnaas Rehabilitation Hospital, Nesoddtangen, Norway; Curato A/S (F.G.L.), Oslo, Norway; Department of Medical Genetics (B.P.), Oslo University Hospital, Ullevaal, Oslo, Norway; and Faculty Divison Rikshospitalet (R.L., S.R.-H., O.G., H.-J.S.), University of Oslo, Oslo, Norway.

Please address correspondence to Rigmor Lundby, MD, Department of Radiology, Oslo University Hospital, Rikshospitalet, Sognsvannsveien 20, N0-0027 0slo, Norway; e-mail: rigmor.lundby@medisin.uio.no

Indicates article with supplemental on-line table.

DOI 10.3174/ajnr.A1620 cortex of the pedicles and laminae of the vertebrae; widening of the neural foramina; or an anterior meningocele."

A more recent definition of $\mathrm{DE}$ is widening of the dural sac or spinal nerve root sleeves, usually associated with bony erosions of the posterior vertebral body. ${ }^{3}$

Although a number of articles about MFS have presented methods on how to assess DE by using conventional $\mathrm{x}$-ray films, CT, and MR imaging, ${ }^{4-7}$ no gold standard for the diagnosis of DE has emerged. A number of radiologic features of DE have been suggested, including anterior meningoceles, herniations of the dura along the nerve root sleeves, ${ }^{4}$ wider dural sac at $\mathrm{S} 1$ compared with L4, ${ }^{4}$ and elevated dural sac ratios (DSR) (ie, the ratio between the dural sac diameter [DSD] and the vertebral body diameter [VBD] at the same level). ${ }^{6}$

Although some MFS studies have included controls with respect to $\mathrm{DE}$, these control groups, with 1 exception, tended to be small; they have not been sex- and age-matched; and they have included patients with low back symptoms. ${ }^{6-10}$ The impact of DE in the diagnostic process of MFS has been discussed in several articles. ${ }^{4,6,8-13}$ However, in most studies describing cohorts of patients with MFS, the patients have not been examined for $\mathrm{DE},{ }^{14}$ in spite of the fact that $\mathrm{DE}$ is seen in a high percentage of patients with this syndrome. The incidence of DE in patients fulfilling the Ghent criteria (Ghent-positive patients) has been reported to be from $63 \%$ to $92 \%$. $^{6-8,11,13}$

$\mathrm{DE}$ is a major diagnostic feature for MFS but has also been reported in patients with scoliosis, neurofibromatosis, ${ }^{15}$ ankylosing spondylitis, ${ }^{16,17}$ and Ehlers-Danlos syndrome, ${ }^{7}$ as well as following trauma. Recently, DE has been found in persons with mutations in the genes TGFBR1 and TGFBR2, some of these individuals fulfilling the Ghent criteria, and some not. ${ }^{18-20}$ Thus, it may be argued that DE is a sign of inherited connective tissue disorders. In line with this, DE was not identified as an incidental finding in a study concerning MR imaging of the lumbar spine in persons without back pain. ${ }^{21}$ 
By investigating the morphology of the lumbosacral spine of 105 adults with MFS or suspected MFS and 101 sex- and age-matched controls, we aimed, in this case control study, to establish the prevalence of DE in a cohort of patients fulfilling the Ghent criteria for MFS and to find the best criteria for assessment of DE.

\section{Materials and Methods}

The study was approved by the regional medical ethics committee; and for the purpose of informed consent, only patients older than 18 years of age were included.

\section{Study Population}

The participants in the study were recruited either by an invitation letter sent to the 134 individuals older than 18 years of age registered in a national resource center as having MFS; by advertisement in the Journal of the National Association of Marfan Syndrome and Other Marfanlike Conditions, asking for persons having the diagnosis of MFS; or through invitations distributed in the Department of Thoracic and Cardiovascular Surgery at our hospital to patients suspected of having MFS. Age was the only exclusion criterion.

A total of 109 individuals gave their informed consent for participation. One patient died before the study started, 1 was not able to attend while living abroad, and 2 participants withdrew. Consequently, the study population consisted of 105 persons (67 women; $20-69$ years of age; mean, $41.3 \pm 12.8$ years; and 38 men; $19-62$ years of age; mean, $36.5 \pm 10.8$ years). Before inclusion, 90 had been given a diagnosis of MFS and 15 were suspected of having the diagnosis. All participants were white.

Everyone in the study group was assessed for all parts of the Ghent criteria $^{1,2}$ by the same group of physicians. The assessment included sequencing of the entire coding region of the gene FBN1 and a search for large deletions or duplications. ${ }^{22,23}$

\section{Control Population}

The control subjects were chosen from the pool of patients in the radiologic archive (PACS) of the department of radiology on the basis of the following criteria: sex- and age-matched asymptomatic persons with respect to the lumbosacral spine and without any known connective tissue disease or compression fractures, screened with MR imaging for malignancy in the lumbosacral spine, but with no evidence of malignant disease in this area. The controls included 101 subjects, 64 women ( $18-65$ years of age; mean, $39.6 \pm 12.9$ years) and 37 men ( $18-70$ years of age; mean, $35.7 \pm 12.3$ years).

\section{MR Imaging or CT of Patients in the Study Population}

MR imaging of the lumbosacral spine was performed unless contraindicated, when CT examinations were obtained instead.

MR imaging was performed by using a 1.5T unit (Magnetom Vision Plus; Siemens, Erlangen, Germany). T1-weighted (TR/TE $=375 / 9 \mathrm{~ms})$ and T2-weighted $(\mathrm{TR} / \mathrm{TE}=3500 / 120 \mathrm{~ms})$ turbo spin-echo sequences were obtained in the sagittal plane with 4-mm section thickness. The T2-weighted sequence was repeated in the coronal plane and in 5 angulated axial planes parallel to the 5 lumbar intervertebral disks.

Axial CT images with 5-mm section thickness were obtained on a ProSpeed SX scanner (GE Healthcare, Milwaukee, Wis) from T12 through the lumbar and sacral regions in the 6 patients in whom CT examinations were performed.

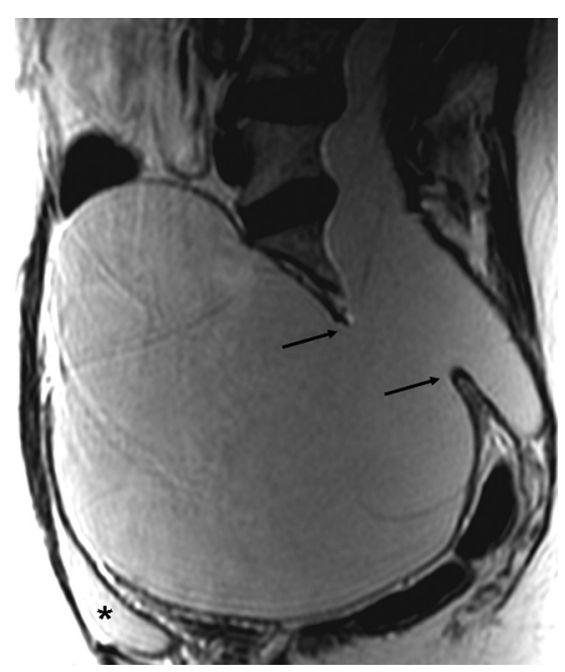

Fig 1. Sagittal T2-weighted MR image of a patient with MFS showing a huge anterior meningocele herniating into the pelvis through a large sacral defect (arrows). The urinary bladder (asterisk) is compressed anteriorly.

\section{MR Imaging of Controls}

All MR imaging studies in the control group were performed with a 1.5T unit (Signa, GE Healthcare) with sagittal and coronal T1weighted fast spin-echo sequences $(\mathrm{TR} / \mathrm{TE}=500 / 9-13 \mathrm{~ms})$ and sagittal and coronal short-inversion-time inversion recovery sequences $(\mathrm{TR} / \mathrm{TI} / \mathrm{TE}=4300 / 150 / 34 \mathrm{~ms})$. Section thickness and gap were 4 and $0.5 \mathrm{~mm}$ for sagittal images and 7 and $1 \mathrm{~mm}$ for coronal images, respectively.

\section{Evaluation}

The control and study populations were compared in aggregate. Consensus readings of all imaging studies of the patients with MFS were performed by 1 neuroradiologist (R.L.) unaware of the clinical information and 1 specialist in rehabilitation medicine (S.R.-H.) aware of the clinical information. Consensus interpretations of the controls were obtained by 2 neuroradiologists (R.L. and J.K.H.). A third radiologist (S.S.) assessed all the above studies for interobserver agreement.

\section{Measurements and Definitions}

An anterior sacral meningocele was diagnosed when a herniation of the dural sac through a defect in the anterior surface of the sacrum was $\operatorname{seen}^{24}$ or when the sacral meninges were herniating anteriorly into the pelvis through a widened foramen, which may be a finding secondary to herniation of the meninges anteriorly (Fig 1). ${ }^{25}$

Herniation of a nerve root sleeve, a lateral meningocele, was considered present when the nerve root sleeve was wide throughout the intervertebral foramen and ended in a pouch (Fig 2).

On sagittal MR images, anteroposterior (AP) VBD was measured halfway between the superior and inferior endplates and at the cranial and caudal endplate perpendicular to the long axis of the vertebral bodies of L3, L4, L5, and S1 (Fig 3A). The craniocaudal heights of the vertebrae were measured anteriorly, halfway between the anterior and dorsal margin of the vertebrae, and dorsally at the same levels (Fig $3 B$ ). DSDs were measured halfway between the superior and inferior endplates from T12 through the lumbar and sacral regions to the caudal end of the dural sac (Fig $3 B$ ). All of these measurements were obtained on the midline sagittal image (Fig 3A, -B). DSRs (DSR = $\mathrm{DSD} / \mathrm{VBD}$ at the midcorpus level) were calculated at levels L3-S1, and 


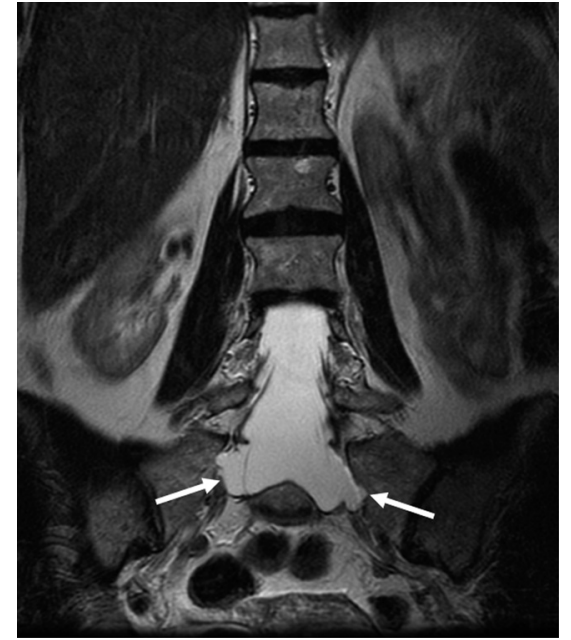

Fig 2. Coronal T2-weighted MR image showing lateral meningoceles/herniations (arrows) of the nerve root sleeves at level S1 in a patient with MFS.

the presence of a DSD at level S1 or below greater than DSD at level L4 (DSD sacrum > DSDL4) was noted.

Scalloping was assessed in all the lumbar and the first sacral segments; it was considered present when the posterior vertebral margin halfway between the superior and inferior endplates was located $>2$ $\mathrm{mm}$ anterior to a line drawn from the upper to the lower posterior margin of the vertebral body.

The presence of perineural cysts (Tarlov cysts) was assessed. We considered a cystic dilation containing fluid with spinal fluid signal intensity along the nerve root to be a perineural cyst when a septum through the neural foramen was present and/or the foramen was not widened. This visual definition of perineural cysts was made according to Acosta et al. ${ }^{26}$

For the individual patient, the diagnosis of DE was based on the presence of $\geq 1$ of the following findings: anterior sacral meningocele, $\geq 1$ dural sac nerve root sleeve herniations, DSD at level S1 or below greater than the DSD at level L4 (DSD sacrum $>$ DSDL4), DSR at L5 $>0.48$, and DSR at S1 $>0.57 .4,6,9$

In addition to diagnosing DE, we identified the level of the caudal end of the dural sac, the tip of the conus medullaris, and any disk herniations.

\section{Statistics}

For continuous data, differences in the mean between the 4 study groups were analyzed with 1-way analysis of variance and the Tukey post hoc tests for multiple comparisons. Statistical analysis of the DSD between the groups was adjusted for sex and age by using a general linear model. The Pearson $\chi^{2}$ test was used for categoric data. Odds ratios were calculated for nerve root sleeve herniations. Interobserver agreement was analyzed by constructing Bland-Altman plots or with $\kappa$ statistics. To assess how DSR could be used to differentiate Ghent-positive patients independent of the dura (group 1) from controls (group 4), we constructed receiver operating characteristic (ROC) curves. A cutoff value for DSR for diagnosis of MFS with a given sensitivity and specificity was derived from the ROC curve. Significance level was set to $5 \%$. All statistical analyses were performed by the Statistical Package for the Social Sciences, Version 16.0 (SPSS, Chicago, Ill).

\section{Results}

The study population was divided into 3 groups through the clinical assessment for MFS: 1) persons fulfilling the Ghent criteria independent of DE (Ghent independent of the dura, $n=73)$, 2) persons fulfilling the Ghent criteria dependent on DE (Ghent dependent on the dura, $n=14$ ), and 3) persons suspected of having MFS, but not fulfilling the Ghent criteria (not Ghent, $n=18)$. The control population $(n=101)$ constituted group 4 (Tables 1 and 2).

Anterior meningoceles (Tables 1 and 2) (Fig 1) were found in Ghent-positive patients only. They were present in 37\% in group 1 and 14\% in group 2 . The sensitivity of this finding in diagnosing MFS was $37 \%$ and the specificity, $100 \%$.

Herniations of the nerve root sleeve were frequently present in Ghent-positive patients (Tables 1 and 2) (Fig 2). They were most commonly found at levels S1 and S2 (Fig 2). The presence of nerve root sleeve herniation at $\geq 1$ level had a sensitivity of $72.6 \%$ (95\% confidence interval [CI], 67.6\%$77.6 \%)$ and a specificity of $99.0 \%$ (95\% CI, 95.4\%-99.8\%) in diagnosing MFS. Most Ghent-positive patients had $>1$ herniation of the nerve root sleeve, whereas the single control person found to have herniation of the nerve root sleeve had only 1 and none from group 3 had $>1$ herniation.

A DSD at S1 or below, larger than the DSD at L4, was found in a high proportion of Ghent-positive patients (Tables 1 and 2 ) but was a rare finding in Ghent-negative patients or controls. This difference was statistically significant $(P<.001)$. A DSD sacrum $>$ DSDL4 had a sensitivity of $55.1 \%(95 \%$ CI, $48.2 \%-59.0 \%$ ) and a specificity of $95.0 \%$ (95\% CI, $90.4 \%-$ $97.8 \%$ ) in diagnosing MFS. One person from the control group had a DSD sacrum $>$ DSDL4 difference of $3 \mathrm{~mm}$; the other 5 patients positive for this sign in the control group had a 1-mm difference between levels S1 and L4.

At the 3 levels L4-S1, the Ghent-positive patients (groups 1 and 2) had significantly larger mean DSRs than the Ghentnegative patients and controls (groups 3 and 4$)(P<.001)$. At level L3, the difference in mean DSR between groups 1 and 4 was not significant. There were no significant differences in mean DSR between groups 1 and 2 or between groups 3 and 4 at any level (L3-S1).

Mean DSRs in Ghent-positive patients independent of DE (group 1) were compared with mean DSRs in the controls (group 4) at the 4 levels L3-S1 by using ROC analysis (Fig 4). The area under the curve for S1 was 0.88 (95\% CI, $0.82 \%$ $0.94 \%$ ) and was the largest area at the different levels. On the basis of the ROC analysis, a cutoff value of 0.59 for DSR at level S1 was suggested, giving a sensitivity of $70.5 \%$ and a specificity of $92.7 \%$ as a marker for MFS.

The combined signs of DE as described in the "Materials and Methods" section, including cutoff values of DSR at levels L5 and S1 of Oosterhof et al, ${ }^{6}$ indicated the presence of DE in $91 \%$ of all the Ghent-positive patients ( 79 out of 87 ) and in $89 \%$ of the Ghent-positive patients independent of DE (Table 2 ). Twenty-four percent of the controls were also given a diagnosis of DE on the basis of these criteria (Table 2). Four of 73 patients in group 1 fulfilled elevated DSR only as a sign of DE. Similarly, 4 of 14 Ghent-positive patients dependent on DE relied on an elevated DSR for their diagnosis. Exchanging the DSR cutoff values of Oosterhof et $\mathrm{al}^{6}$ with the cutoff value at S1 (0.59) suggested by our ROC analysis resulted in a loss of MFS 

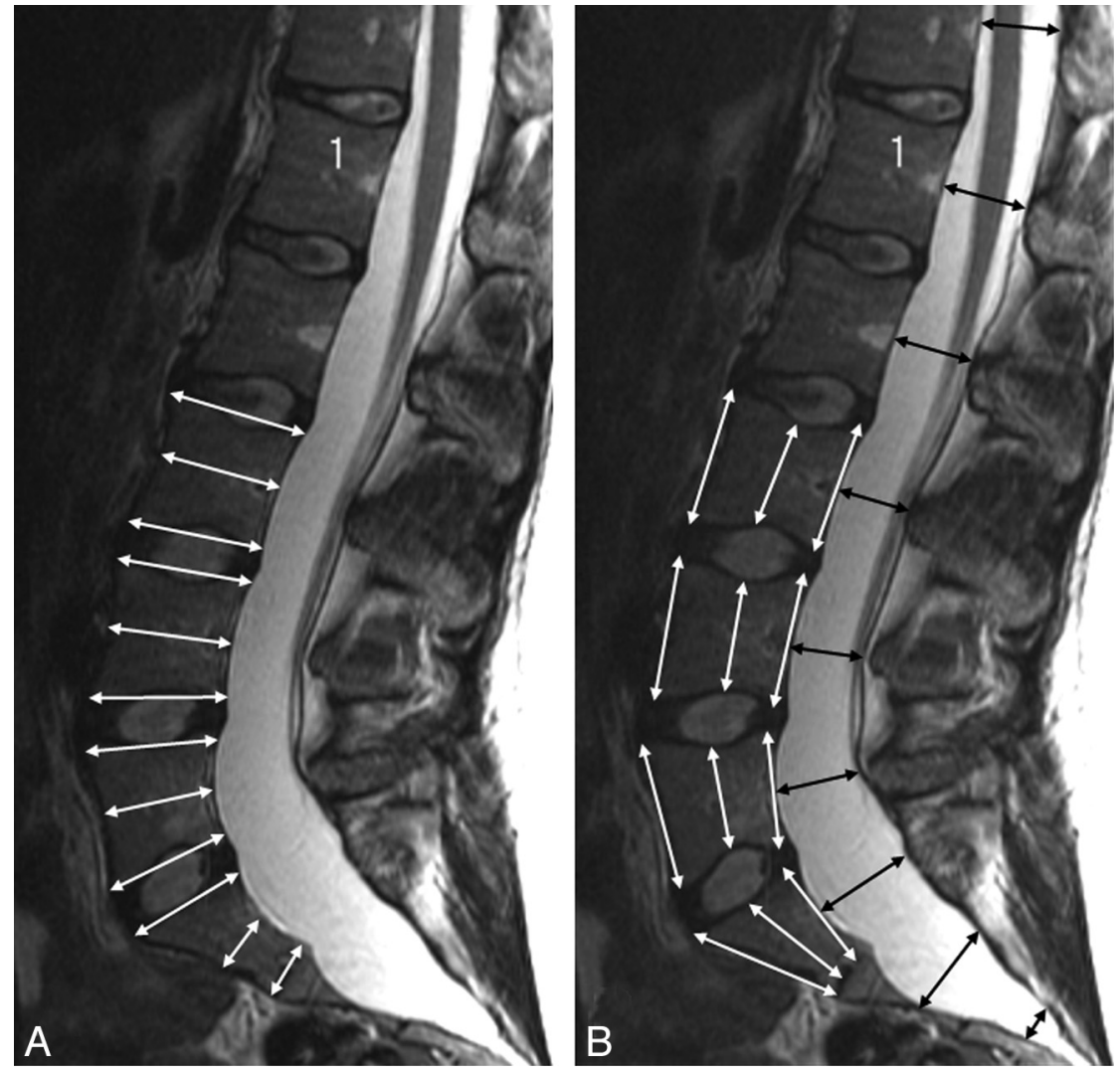

Fig 3. $A$ and $B$, T2-weighted sagittal MR images of a patient with MFS showing craniocaudal and AP measurements of the vertebral bodies (white arrows, $A$ and $B$ ) and AP measurements of the dural sac (black arrows, B). Scalloping is present at levels $L 5, S 1$, and S2.

diagnoses in 2 of the 14 Ghent-positive patients in group 2 and reduced the number of persons fulfilling DE criteria in groups 1 and 2 (Table 2). This higher DSR cutoff value reduced the number of presumed false-positive diagnoses of DE in the control group from $24(24 \%)$ to 9 (9\%) (Table 2).

Scalloping was present significantly more often in Ghent-

\begin{tabular}{|c|c|c|c|c|c|}
\hline Characteristics & $\begin{array}{c}\text { Ghent Independent } \\
\text { of } \mathrm{DE} \\
(n=73)\end{array}$ & $\begin{array}{c}\text { Ghent Dependent } \\
\text { on DE } \\
(n=14)\end{array}$ & $\begin{array}{c}\text { Not Fulfilling } \\
\text { Ghent } \\
(n=18)\end{array}$ & $\begin{array}{c}\text { Control } \\
(n=101)\end{array}$ & $P$ Value \\
\hline Age (mean \pm SD) & $39.4(12.2)$ & $40.6(17.1)$ & $36.1(13.4)$ & $39.6(12.9)$ & .74 \\
\hline Females (No.) (\%) & $45(61.6)$ & $11(78.6)$ & $11(61.6)$ & $64(63.4)$ & .68 \\
\hline DSR L3 (mean \pm SD) & $0.50^{\mathrm{b}, \mathrm{c}}(0.11), n=69$ & $0.53^{\mathrm{c}}(0.06)$ & $0.43^{\mathrm{a}}(0.08), n=14$ & $0.45^{\mathrm{a}, \mathrm{b}}(0.07)$ & $<.001$ \\
\hline DSR L4 (mean \pm SD & $0.50^{\mathrm{b}}(0.12), n=72$ & $0.51^{\mathrm{b}}(0.07)$ & $0.41^{\mathrm{a}}(0.06)$ & $0.43^{\mathrm{a}}(0.07)$ & $<.001$ \\
\hline DSR L5 (mean \pm SD) & $0.57^{\mathrm{b}}(0.18), n=68$ & $0.65^{b}(0.20)$ & $0.39^{\mathrm{a}}(0.09)$ & $0.42^{\mathrm{a}}(0.08)$ & $<.001$ \\
\hline DSR S1 (mean \pm SD) & $0.94^{\mathrm{b}}(0.69), n=61$ & $1.02^{b}(0.77), n=13$ & $0.40^{\mathrm{a}}(0.12), n=12$ & $0.41^{\mathrm{a}}(0.13), n=96$ & $<.001$ \\
\hline DSD sacrum > DSD L4 & $38(55.1 \%), n=69$ & $4(28.6 \%)$ & $1(5.6 \%)$ & $5(5.0 \%)$ & $<.001$ \\
\hline \multicolumn{6}{|l|}{ Herniation of nerve root sleeves } \\
\hline Present in $\geq 1$ level (No.) (\%) & $53(72.6 \%)$ & $10(71.4 \%)$ & $2(11.1 \%)$ & $1(1 \%)$ & $<.001$ \\
\hline Present in level L5 & $9(12.3 \%)$ & $0(0 \%)$ & $0(0 \%)$ & $0(0 \%)$ & $<.001$ \\
\hline Present in level S1 & $35(47.9 \%)$ & $8(57.1 \%)$ & $2(11.1 \%)$ & $0(0 \%)$ & $<.001$ \\
\hline Present in level S2 & $48(65.8 \%)$ & $8(57.1 \%)$ & $1(5.6 \%)$ & $1(1 \%)$ & $<.001$ \\
\hline Present in level S3 & $30(41.1 \%)$ & $5(35.7 \%)$ & $0(0 \%)$ & $0(0 \%)$ & $<.001$ \\
\hline Present in level $\$ 4$ & $8(11 \%)$ & $1(7.1 \%)$ & $0(0 \%)$ & $0(0 \%)$ & $<.004$ \\
\hline $\begin{array}{l}\text { Odds ratio, } \geq 1 \text { herniation of the } \\
\text { nerve root sleeve }\end{array}$ & 265 (34-2029) & $250(25-2458)$ & $12.5(1-145)$ & ref & $<.001$ \\
\hline Anterior meningocele & $27(37 \%)$ & $2(14 \%)$ & 0 & 0 & $<.001$ \\
\hline Scalloping L1-S1 $\geq 1$ level & $44(61.1 \%), n=72$ & $8(42.9 \%)$ & $1(5.6 \%)$ & $4(4 \%), n=100$ & $<.001$ \\
\hline Perineural cysts & $11(15.1 \%)$ & $1(7.1 \%)$ & $1(5.6 \%)$ & $8(7.9 \%)$ & .387 \\
\hline Disk herniation beneath T12 & $5(7 \%), n=69$ & $1(7 \%)$ & $2(11 \%)$ & $6(6 \%)$ & .890 \\
\hline \multicolumn{6}{|l|}{ Caudal end of dural sac } \\
\hline L5, L5/S1, S1 & $10(14.5 \%)$ & $0(0 \%)$ & $9(50 \%)$ & $22(21.8 \%)$ & $<.001$ \\
\hline S2 & $26(37.7 \%)$ & $9(64.3 \%)$ & $8(44.4 \%)$ & $68(67.3 \%)$ & $<.001$ \\
\hline S3, S4, S5 & $33(47.8 \%), n=69$ & $5(35.7 \%)$ & $1(5.6 \%)$ & $11(10.9 \%)$ & $<.001$ \\
\hline
\end{tabular}

Note:-DE, dural ectasia; DSR, dural sac ratio; DSD, dural sac diameter.

* Study groups not containing similar letters (a, b, c, or d) for means were statistically different at the $5 \%$ level. Where $n$ is specified in a cell, there were some observations missing and $n$ is the actual number of observations. Missing values are due to incomplete anatomic coverage with CT, extreme scoliosis at MR, or the dural sac ending above the missed level. 


\begin{tabular}{|c|c|c|c|c|}
\hline \multirow[b]{2}{*}{ Criteria } & \multicolumn{4}{|c|}{ Fulfilling Ghent (No.) (\%) } \\
\hline & $\begin{array}{l}\text { Independent of DE } \\
\quad(n=73)\end{array}$ & $\begin{array}{l}\text { Dependent on DE } \\
\quad(n=14)\end{array}$ & $\begin{array}{l}\text { Suspected MFS, } \\
\text { Not Fulfilling Ghent } \\
(n=18)\end{array}$ & $\begin{array}{l}\text { Controls } \\
(n=101)\end{array}$ \\
\hline 1) Anterior meningocele & $27(37)$ & $2(14)$ & $0 \%$ & $0 \%$ \\
\hline 2) DSD sacrum > DSD L4 & $38(52)$ & $4(29)$ & $1(6)$ & $5(5)$ \\
\hline 3) Herniations of $\geq 1$ nerve root sleeves & $53(73)$ & $10(71)$ & $2(11)$ & $1(1)$ \\
\hline 4) DSR L5 > $0.48\left(\right.$ Oosterhof et $\left.\mathrm{al}^{6}\right)$ & $41(56)$ & $13(93)$ & $1(6)$ & $19(19)$ \\
\hline 5) DSR S1 > 0.57 (Oosterhof et al ${ }^{6}$ ) & $45(62)$ & $11(85)$ & $1(6)$ & $12(12)$ \\
\hline Presence of $\geq 1$ of above findings & $65(89)$ & $14(100)$ & $3(17)$ & $24(24)$ \\
\hline Only DSR L5 $>0.48$ and/or $S 1>0.57$ & $4(5)$ & $4(29)$ & $1(6)$ & $18(18)$ \\
\hline 6) DSR S1 > 0.59 (current study) & $43(59)$ & $11(85)$ & $1(6)$ & $7(7)$ \\
\hline Presence of DE replacing criteria 4 and 5 with 6 & $63(86)$ & $12(86)$ & $3(17)$ & $9(9)$ \\
\hline
\end{tabular}

Note:-MES indicates Marfan Syndrome.

* Expressed as number and percentage.

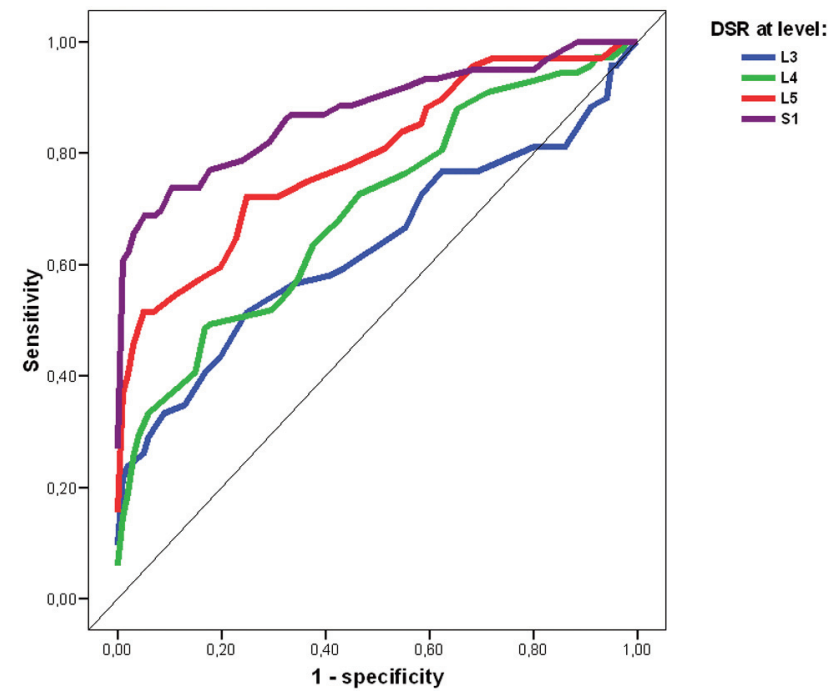

Fig 4. ROC curves of DSR at levels L3-S1. Graph shows sensitivity versus (1-specificity) of DSR as a marker of MFS at levels L3-S1 when comparing Ghent-positive patients independent of DE and controls.

positive patients compared with Ghent-negative persons and controls $(P<.001)$ (Table 1$)$.

No significant differences between the 4 groups were found with respect to perineural cysts, frequency of disk herniations, or the level of the conus.

A position of the caudal end of the dural sac distal to level S2 (ie, at levels S3-S5) was seen significantly more often in Ghent-positive patients than in Ghent-negative patients and controls $(P<.001)$.

Measures of height and depth of vertebrae were adjusted for sex (On-line Table 1). The S1 vertebral body was found with significantly reduced AP diameters in patients with MFS compared with others, as was the caudal part of the L5 vertebra. There were a few significant differences in heights between the 4 groups, but with no consistent pattern. DSD was significantly larger in Ghent-positive patients than in group 3 and controls when adjusting for sex and age at levels T12 all way down to level S2; but at lower levels, there were few persons with persisting dural sac in groups 3 and 4 . Thus, no significance level could be calculated.

\section{Interobserver Study}

Interobserver agreement analysis gave a $\kappa$ for herniation of nerve root sleeve at $\geq 1$ level at 0.88 (95\% CI, 0.79-0.93) and, for assessment of herniation at a specified level, 0.23 at level L5, 0.37 at level S1, and 0.32 at level S2. The interclass correlation coefficient for DSR at level L4 was 0.82 (95\% CI, 0.770.86); at level L5, 0.92 (95\% CI, 0.90-0.94); and at level S1, 0.97 (95\% CI, 0.96-0.98). Interobserver agreement $\kappa$ for DSD at level S1 or below larger than DSD at level L4 was 0.77 (95\% CI, 0.65-0.85).

\section{Discussion}

DE is a major criterion for MFS and is present in most patients, also those being Ghent-positive on the basis of other major criteria. The diagnosis of DE is especially important for patients dependent on DE to become Ghent-positive. A total of $91 \%$ of all Ghent-positive patients in our study was found to have DE on the basis of anterior or lateral meningoceles, DSD sacrum $>$ DSD L4, or the cutoff values at level L5 and S1 of Oosterhof et al. ${ }^{6}$ Fourteen of 87 patients with MFS (16\%) were dependent on DE to fulfill the Ghent criteria, ${ }^{1}$ underscoring the importance of assessing the dural sac in individuals investigated for MFS. ${ }^{2}$ The dura is 1 of 5 organ systems with major criteria of MFS in the Ghent nosology. Although the dural sac is a soft-tissue structure, DE has been defined to include bony changes of the spine, such as thinning of the cortex of the posterior vertebral elements, widening of the neural foramina, and vertebral scalloping in addition to the presence of a patulous dural sac and anterior meningoceles. ${ }^{1,27} \mathrm{MR}$ imaging, like $\mathrm{CT}$ and conventional radiographs, identifies the secondary osseous changes associated with $\mathrm{DE}$, but MR imaging also allows the direct visualization of soft tissues of the entire spine. Sagittal images best identify the AP spinal canal and vertebral body diameters, whereas additional information may be obtained from axial and coronal images. The latter are of particular importance to characterize lateral or anterior meningoceles.

CT in our study was inferior in quality compared with MR imaging, in particular because we had a 5-mm section thickness only and no reformatting, as would have been possible with thinner sections.

DE may be evaluated either qualitatively or quantitatively, and different methods have been described. Some recent reports about DE in MFS have postulated that quantitative signs 
of DE have major advantages over qualitative assessments because cutoff values can be used more uniformly than qualitative signs. ${ }^{6,9}$ We found qualitative signs very useful because, for instance, nerve root sleeve herniations were present in many of our Ghent-positive patients independent of dura and 10 of our patients would not have received the diagnosis of DE if nerve root sleeve herniation had not been a sign of DE.

Anterior meningocele is a strong qualitative indicator of MFS and has been used as a sign of DE in many studies and articles. ${ }^{1,4}$ We found no anterior meningoceles in groups 3 and 4 (specificity, $100 \%$ ), but $37 \%$ of group 1 had anterior meningoceles. The interobserver correlation coefficient for anterior meningocele was 0.78 . Thus, an anterior meningocele is a very specific finding but is seen only in extensive DE and is, therefore, not a very sensitive sign of DE.

Herniation of nerve root sleeves has been used as a diagnostic feature of DE by different authors and has been termed "lateral meningoceles," "radicular cysts," and "protrusion of dura outside the neural canal" in previous publications. ${ }^{8,27-29}$ Herniation of the nerve root sleeves was frequently found at $\geq 1$ level in persons with MFS, but rarely in controls. The odds ratio was significantly higher in Ghent-positive patients versus controls with respect to herniations at $\geq 1$ level (Table 1 ). Interobserver agreement was high $(\kappa=0.88)$, indicating that herniations can be assessed relatively consistently. It proved more difficult, however, to determine the correct level for herniations, as illustrated by the low $\kappa$ values for assessment of this finding: 0.23 at level L5, 0.37 at level S1, and 0.32 at level S2. This was due to the fact that a single herniation could extend for several vertebral levels. Coronal imaging was an important help in determining the correct levels and in discriminating herniations from perineural cysts. No significant differences were found in the prevalence of perineural cysts between the different groups. These cysts may, however, be difficult to separate from herniations of the nerve root sleeves, a well-known point of discussion for many years. ${ }^{26}$

DSD at level S1 or below larger than DSD at level L4 was used as a quantitative criterion of DE and could be assessed with high interobserver agreement $(\kappa=0.77)$. This finding was present significantly more often in our Ghent-positive population than in "not Ghent" and controls, and it has also been shown to be a useful marker for DE in earlier studies. ${ }^{4,9}$ This difference in DSD at levels L4 and S1 or below was, however, also found in 5\% of the controls ( 5 subjects). Only 1 of these had $>1 \mathrm{~mm}$ difference in DSD. Increasing the border for pathology of DSD sacrum $>$ DSD L4 from 0 to $1 \mathrm{~mm}$ would minimize the presumed false-positives, but this would also reduce the sensitivity of this finding for MFS to $52.5 \%$.

DSR has been used to assess DE in recent studies. Oosterhof et $\mathrm{al}^{6}$ were the first to describe DE in this quantitative manner only. They concluded that a combination of DSR above a given cutoff value at level L3 and S1 could be used to identify MFS with 95\% sensitivity and 98\% specificity. Their method has been discussed and tested in later studies, but similar results have not been obtained. ${ }^{8,12}$ Habermann et $\mathrm{al}^{9}$ found a difference in DSR between patients with MFS and controls only at level L5 and S1; but with an optimal cutoff value of 0.51 at level S1, they found a sensitivity of $56 \%$ and a specificity of $65 \%$. Most important, this was a study of children, youths, and young adults, and the authors suggested that some of the dif- ferences in cutoff values and accuracy were secondary to the age differences in the 2 studies. Weigang et al ${ }^{13}$ detected DE in $94 \%$ of patients with MFS and in $44 \%$ without MFS when they followed the methods and cutoff values of Oosterhof et al. ${ }^{6}$

Using the cutoff values from Oosterhof et al, ${ }^{6}$ we found that our controls had DSR values suggesting DE in $12 \%$ at level S1 and in $19 \%$ at level L5 (Table 2), indicating that these cutoff values are too low. DSR at level L3 had no discriminating value for the presence of MFS because we found no significant differences between Ghent-positive patients independent of DE and the controls. In our study, the levels at which measurement of DSR best indicated the presence of MFS were S1 and L5 (Fig 4). These findings are supported by the those of Habermann et $\mathrm{al}^{9}$ in younger patients. At level S1, we suggest a cutoff level at 0.59 on the basis of our ROC curve (Fig 4). Even with this cutoff value, $9 \%$ of the controls were given the diagnosis of DE. However, we prefer not to decrease sensitivity (70.5\%) even more and, therefore, recommend this cutoff value so far.

At the 3 levels L4-S1, the Ghent-positive patients (groups 1 and 2) had significantly larger mean DSRs than the Ghentnegative patients and controls (groups 3 and 4) as stated in the "Results" section.

Scalloping is another sign used for assessment of DE, especially when only a lateral radiograph of the spine is available. ${ }^{4}$ It has been assigned as a minor criterion for DE, also on CT and MR imaging. Habermann et $\mathrm{al}^{9}$ included scalloping at level L5 and S1 to evaluate if this was a useful marker for DE but found no differences in scalloping between patients with MFS and controls in their population of children, adolescents, and young adults. Ahn et $\mathrm{al}^{4}$ used level S1 only for assessing scalloping as a sign of DE and found this to be a useful but minor criterion for DE. We found significantly higher presence of scalloping at $\geq 1$ level in Ghent-positive patients than in others. It can, therefore, be argued that spinal skeletal changes should be included in the criteria for DE as well as changes in dura mater. However, we chose to use the softtissue dural changes and not the skeletal ones as decisive when assessing DE because the positive finding of a widened dural sac is more easily assessed with MR imaging than is scalloping.

The dural sac was found to end at a lower level in patients with MFS than in patients in groups 3 and 4, supporting the theory that development of DE is influenced by hydrostatic pressure. $^{30}$

It has been claimed that patients with MFS have higher and narrower vertebral bodies than healthy individuals. ${ }^{31} \mathrm{We}$ found no unilateral pattern that strengthens this theory, except for lower AP diameters caudally in Ghent-positive patients (On-line Table 1).

The caudal part of the L5 vertebral body and the S1 vertebral body had significantly shorter AP diameters in patients with MFS than in others. Remodelling due to a combination of high hydrostatic pressure at the bottom of the spinal canal and a weakened dural sac in patients with DE seems a likely explanation. ${ }^{9,30}$

\section{Conclusions}

Our case-control study shows that assessment of DE should be based on both qualitative and quantitative findings to avoid false-positive results. DE was found in $86 \%$ of our Ghentpositive patients independent of DE. Only a few of our Ghent- 
positive patients were dependent on elevated DSR to obtain the diagnosis of DE. We suggest that a diagnosis of DE should be based on the presence of at least 1 of the following criteria: 1) anterior meningoceles or nerve root sleeve herniation, 2) DSD at level S1 or below larger than DSD at level L4, and 3) DSR at level $\mathrm{S} 1>0.59$. MR imaging of the spine is encouraged to identify DE and thus strengthen a potential diagnosis of MFS.

\section{References}

1. De Paepe A, Devereux RB, Dietz HC, et al. Revised diagnostic criteria for the Marfan syndrome. Am J Med Genet 1996;62:417-26

2. Rand-Hendriksen S, Lundby R, Tjeldhorn L, et al. Prevalence data of all Ghent features in a cross sectional study of 87 adults with proven diagnosis Marfan syndrome. Eur J Hum Genet 2009 Mar 18. [Epub ahead of print]

3. Avivi E, Arzi H, Paz L, et al. Skeletal manifestations of Marfan syndrome. Isr Med Assoc J 2008;10:186-88

4. Ahn NU, Sponseller PD, Ahn UM, et al. Dural ectasia in the Marfan syndrome: MR and CT findings and criteria. Genet Med 2000;2:173-79

5. Ahn NU, Nallamshetty L, Ahn UM, et al. Dural ectasia and conventional radiography in the Marfan lumbosacral spine. Skeletal Radiol 2001;30:338-45

6. Oosterhof T, Groenink M, Hulsmans FJ, et al. Quantitative assessment of dural ectasia as a marker for Marfan syndrome. Radiology 2001;220:514-18

7. Villeirs GM, Van Tongerloo AJ, Verstraete KL, et al. Widening of the spinal canal and dural ectasia in Marfan's syndrome: assessment by CT. Neuroradiology 1999;41:850-54

8. Fattori R, Nienaber CA, Descovich B, et al. Importance of dural ectasia in phenotypic assessment of Marfan's syndrome. Lancet 1999;354:910-13

9. Habermann CR, Weiss F, Schoder V, et al. MR evaluation of dural ectasia in Marfan syndrome: reassessment of the established criteria in children, adolescents, and young adults. Radiology 2005;234:535-41

10. Knirsch W, Kurtz C, Haffner N, et al. Dural ectasia in children with Marfan syndrome: a prospective, multicenter, patient-control study. Am J Med Genet A 2006;140:775-81

11. Ahn NU, Sponseller PD, Ahn UM, et al. Dural ectasia is associated with back pain in Marfan syndrome. Spine 2000;25:1562-68

12. Rose PS, Levy HP, Ahn NU, et al. A comparison of the Berlin and Ghent nosologies and the influence of dural ectasia in the diagnosis of Marfan syndrome. Genet Med 2000;2:278-82

13. Weigang E, Ghanem N, Chang XC, et al. Evaluation of three different measurement methods for dural ectasia in Marfan syndrome. Clin Radiol 2006;61: 971-78

14. Faivre L, Collod-Beroud G, Loeys BL, et al. Effect of mutation type and location on clinical outcome in 1,013 probands with Marfan syndrome or related phenotypes and FBN1 mutations: an international study. Am J Hum Genet 2007; 81:454-66. Epub 2007 Jul 25

15. Gajeski BL, Kettner NW, Awwad EE, et al. Neurofibromatosis type I: clinical and imaging features of Von Recklinghausen's disease. J Manipulative Physiol Ther 2003;26:116-27

16. Ahn NU, Ahn UM, Nallamshetty L, et al. Cauda equina syndrome in ankylosing spondylitis (the CES-AS syndrome): meta-analysis of outcomes after medical and surgical treatments. J Spinal Disord 2001;14:427-33

17. Lan HH, Chen DY, Chen CC, et al. Combination of transverse myelitis and arachnoiditis in cauda equina syndrome of long-standing ankylosing spondylitis: MRI features and its role in clinical management. Clin Rheumatol 2007;26:1963-67

18. Ades LC, Haan EA, Colley AF, et al. Characterisation of four novel fibrillin-1 (FBN1) mutations in Marfan syndrome. J Med Genet 1996;33:665-71

19. Loeys BL, Chen J, Neptune ER, et al. A syndrome of altered cardiovascular, craniofacial, neurocognitive and skeletal development caused by mutations in TGFBR1 or TGFBR2. Nat Genet 2005;37:275-81. Epub 2005 Jan 30

20. Loeys BL, Schwarze U, Holm T, et al. Aneurysm syndromes caused by mutations in the TGF-beta receptor. $N$ Engl J Med 2006;355:788-98

21. Jensen MC, Brant-Zawadzki MN, Obuchowski N, et al. Magnetic resonance imaging of the lumbar spine in people without back pain. $N$ Engl J Med 1994;331:69-73

22. Rand-Hendriksen S, Tjeldhorn L, Lundby R, et al. Search for correlations between FBN1 genotype and complete Ghent phenotype in 44 unrelated Norwegian patients with Marfan syndrome. Am J Med Genet A 2007;143A:1968-77

23. Tjeldhorn L, Rand-Hendriksen S, Gervin K, et al. Rapid and efficient FBN1 mutation detection using automated sample preparation and direct sequencing as the primary strategy. Genet Test 2006;10:258-64

24. Oren M, Lorber B, Lee SH, et al. Anterior sacral meningocele: report of five cases and review of the literature. Dis Colon Rectum 1977;20:492-505

25. Moore K. Anterior sacral meningocele. In: Ross JS, Brant-Zawadzki M, Chen M, et al. Diagnostic Imaging: Spine. Salt Lake City: Amirsys; 2004:44-46

26. Acosta FL Jr, Quinones-Hinojosa A, Schmidt MH, et al. Diagnosis and management of sacral Tarlov cysts: case report and review of the literature. $\mathrm{Neu}$ rosurg Focus 2003; 15:E15

27. Moore KR. Dural dysplasia. In: Ross JS, Brant-Zawadzki M, Chen M, et al, eds. Diagnostic Imaging: Spine. Salt Lake City: Amirsys; 2004;118-20

28. De Paepe A. Dural ectasia and the diagnosis of Marfan's syndrome. Lancet 1999;354:878-79

29. Pyeritz RE, Fishman EK, Bernhardt BA, et al. Dural ectasia is a common feature of the Marfan syndrome. Am J Hum Genet 1988;43:726-32

30. Stern WE. Dural ectasia and the Marfan syndrome. J Neurosurg 1988;69: 221-27

31. Magid D, Pyeritz RE, Fishman EK. Musculoskeletal manifestations of the Marfan syndrome: radiologic features. AJR Am J Roentgenol 1990;155:99-104 